\title{
Effect of Ingesting Resistant Maltodextrin on Postprandial Hyperlipidemia Induced by Fructose in Young Women
}

\author{
Kaori Kuzawa $^{1}$, Akihiro Yoshida ${ }^{2}$, Ikuko Tsukamoto $^{3}$, Masaaki Tokuda ${ }^{3}$, Michitaka Naito ${ }^{1}$ \\ ${ }^{1}$ Division of Nutrition \& Health, School \& Graduate School of Life Studies, Sugiyama Jogakuen University, Nagoya, Japan \\ ${ }^{2}$ Department of Clinical Laboratory, Sakashita Hospital, Nakatsugawa, Japan \\ ${ }^{3}$ Faculty of Medicine, Kagawa University, Miki, Japan
}

Email address:

naito@sugiyama-u.ac.jp (M. Naito)

\section{To cite this article:}

Kaori Kuzawa, Akihiro Yoshida, Ikuko Tsukamoto, Masaaki Tokuda, Michitaka Naito. Effect of Ingesting Resistant Maltodextrin on Postprandial Hyperlipidemia Induced by Fructose in Young Women. Journal of Food and Nutrition Sciences. Vol. 7, No. 3, 2019, pp. 49-55. doi: 10.11648/j.jfns.20190703.12

Received: August 2, 2019; Accepted: August 26, 2019; Published: September 19, 2019

\begin{abstract}
Aim: Our previous study demonstrated that the ingestion of fructose with fat exacerbated and delayed postprandial lipid metabolism (J Atheroscler Thromb 2013; 20: 591). Herein, we investigated the effect of ingesting a water-soluble dietary fiber, resistant maltodextrin (RMD), which has been reported to be effective for ameliorating postprandial glycemia and lipidemia, on fructose-induced postprandial hyperlipidemia in healthy young women. Methods: Healthy young Japanese women with apolipoprotein E3/3 phenotype were enrolled. They underwent 4 test trials in a randomized crossover design: fat cream $(0.35 \mathrm{~g} / \mathrm{kg}$ of fat; F trial), fat cream with RMD ( $5 \mathrm{~g}$; FR trial), fat cream with fructose $(0.5 \mathrm{~g} / \mathrm{kg}$; FFr trial), and fat cream with fructose and RMD (FFrR trial). Blood samples were taken before (0) and at 0.5, 1, 2, 4, and $6 \mathrm{~h}$ after ingestion. Results: The serum glucose and insulin concentrations peaked at $0.5 \mathrm{~h}$ in the FFr and FFrR trials, and no difference was observed between these trials. There was no increase in glucose concentration in the F or FR trials. The serum triglyceride and apolipoprotein B48 concentrations peaked at $4 \mathrm{~h}$ in all trials. In the F and FR trials (but not in the FFr and FFrR trials), the serum triglyceride concentration returned to the fasting level at $6 \mathrm{~h}$. In all trials, the apolipoprotein B48 concentration did not return to baseline at 6 h. Conclusion: Co-ingestion of RMD did not significantly inhibit fructose-induced postprandial hyperlipidemia.
\end{abstract}

Keywords: Resistant Maltodextrin, Fructose, Postprandial Hyperlipidemia, Triglyceride-Rich Lipoprotein, Remnant

\section{Introduction}

In our previous study, the postprandial lipidemia following the ingestion of fructose $(0.5 \mathrm{~g} / \mathrm{kg}$ body mass $)$ in combination with fat cream $(0.35 \mathrm{~g} / \mathrm{kg}$ as fat $)$ was higher than that following the ingestion of glucose [1]. The ingestion of a high-fructose syrup-containing beverage in combination with fat cream also delayed and exacerbated both exogenous and endogenous lipoprotein metabolism, and the ratio of fructose to glucose contained in the beverage was a key factor in the metabolic disturbance when the sugar load was equicaloric [2]. Practically, the ingestion of cola (a high-fructose beverage) in combination with a hamburger (a high-fat diet) was shown to delay postprandial lipidemia compared to the ingestion of a hamburger only, indicating that the fructose contained in the cola delayed and exacerbated postprandial lipidemia [3, 4]. We also showed that smaller amounts of fructose are enough to exacerbate postprandial lipidemia in Japanese women compared to American women [5], suggesting that this problem may be more serious for Japanese people.

Resistant maltodextrin (RMD), a palatable, low-viscous, soluble fiber, has a three-dimensional tree structure, in which $1 \rightarrow 4,1 \rightarrow 6,1 \rightarrow 3$, and $1 \rightarrow 2$ glycosidic bonds are formed during its manufacture. In maltodextrin, in contrast, the $1 \rightarrow 4$ glycosidic bond is predominant, and low percentage of the $1 \rightarrow 6$ glycosidic bond is present [6]. Soluble dietary fibers such as RMD, inulin, and oligofructosaccharide have been shown to have diverse health benefits in the gastrointestinal tract. Colonic bacteria ferment resistant starch and non-starch polysaccharides, which are major components of dietary fiber, to short-chain fatty acids (SCFA), mainly acetate, propionate, and 
butyrate. SCFA stimulate colonic blood flow and fluid and electrolyte uptake. Butyrate is a preferred substrate for colonocytes, and it appears to promote a normal phenotype in these cells. SCFA also keep the $\mathrm{pH}$ in the colonic lumen low. At a lower $\mathrm{pH}$ 6, bile acids are largely protonated and insoluble, and are not taken up by colonocytes [7], and the conversion of primary to secondary bile acids by bacteria is inhibited [8]. In healthy subjects, RMD has been shown to improve colonic motility, fecal characteristics and probiotic (Bifidobacterium) population [9].

In carbohydrate metabolism, RMD is reported to attenuate both postprandial glycemic and insulinemic responses [10]. In lipid metabolism, RMD is also reported to attenuate the response of triglyceride (TG) to ingested fat. In healthy subjects, RMD (5 or $10 \mathrm{~g}$ ) suppressed the postprandial lipidemia induced by a high-fat meal containing $50 \mathrm{~g}$ fat [11]. It was also reported that RMD suppresses the intestinal absorption of lipids by decreasing their micellar solubility and by decreasing the diffusion rate of bile salt micelles [12]. However, RMD did not inhibit lipase activity [13]. Interestingly, RMD suppressed the postprandial rise in blood glucose and insulin levels when consumed with a high-carbohydrate diet, and when it was consumed with a high-fat diet, RMD decreased the rise in the postprandial TG and insulin levels [14]. Postprandial insulin secretion was decreased by RMD in both high-carbohydrate and high-fat diets.

In a longer-term study of healthy subjects, the administration of RMD (5 g) for 10 days suppressed lipid absorption and promoted the excretion of lipid into feces probably by delaying the release of fatty acids from micelles in the lipid absorption process [13]. The 8-week administration of RMD ( $10 \mathrm{~g})$ to women with type 2 diabetes decreased the values of fasting insulin, the homeostasis model assessment for insulin resistance (HOMA-IR), interleukin-6, tumor necrosis factor- $\alpha$, malondialdehyde, and endotoxin [15]. Subjects with metabolic syndrome who took RMD (9 g) at every mealtime for 12 weeks showed decreased values of waist circumference, visceral fat area, fasting blood glucose, HOMA-IR, and serum TG [16].

However, to the best of our knowledge, no studies have examined the effects of RMD on fructose-induced postprandial hyperlipidemia. We therefore examined the effects of RMD, which has been reported to be effective in ameliorating postprandial glycemia and lipidemia, on fructose-induced postprandial hyperlipidemia in healthy young Japanese women. We examined this cohort of women because in the US the highest consumers of high-fructose syrup and sucrose are adolescents and young adults, and in Japan the ingestion of fast food has been reported to be higher among university students than high school students [17]. Although young women are rather resistant to lipid and lipoprotein disturbances, the postprandial TG levels in initially healthy women were reported to be associated with incident cardiovascular events (independent of the levels of other lipids) [18].

\section{Methods}

\subsection{Subjects}

Eleven young Japanese women with normal ovarian cycles and apolipoprotein E phenotype $3 / 3$ were enrolled as participants. All subjects were nonsmokers, had no apparent acute or chronic illnesses, and were not taking any medications or dietary supplements. This study was approved by the Institutional Review Board of the Sugiyama Jogakuen University School of Life Studies (No. 2016-27). The participants provided written informed consent to participate. The procedures were conducted in accordance with the Helsinki Declaration of 1975 as revised in 1983 .

\subsection{Anthropometric and Body Composition Measurement}

Body mass and height were measured according to standard methods. The waist circumference was assessed as the abdominal girth at the level of the umbilicus, and the hip circumference was measured at the level of greater trochanters. The waist-to-hip $(\mathrm{W} / \mathrm{H})$ ratio was calculated. Body composition, including the visceral fat area (VFA), was analyzed using an eight-polar bioelectrical impedance method (InBody720, Biospace, Tokyo, Japan).

\subsection{Experimental Design}

The subjects abstained from consuming caffeine or alcohol during the day before the experiment. Each subject was studied on 4 occasions in a randomized single-blinded crossover design, and they ingested one of the following 4 beverages after a 12-h overnight fast: fat cream (F trial), fat cream with RMD (FR trial), fat cream with fructose (FFr trial), and fat cream with fructose and RMD (FFrR trial). We purchased fat cream (OFTT cream ${ }^{\mathrm{TM}}$ ) from Jomo (Takasaki, Japan), and the subjects ingested $1 \mathrm{~g} / \mathrm{kg}$ body mass $(0.35 \mathrm{~g} / \mathrm{kg}$ as fat) as described [19]. Fructose was ingested as $0.5 \mathrm{~g} / \mathrm{kg}$ body mass. The fructose was dissolved in distilled water and provided as a $10 \%(\mathrm{w} / \mathrm{v})$ solution. In the $\mathrm{F}$ trial, the subjects ingested same amount of water. We purchased the fructose from Kanto Chemical (Tokyo). RMD was provided by Matsutani Chemistry (Itami, Japan). Venous blood samples were obtained before ingestion $(0 \mathrm{~h})$ and at $0.5,1,2,4$, and $6 \mathrm{~h}$ after ingestion. During the test, the subjects avoided exercise and eating, but had free access to water after $1 \mathrm{~h}$. All blood samples were collected while the subjects were in a supine position. There was a 4-week interval between the trial days to minimize the confounding effects of the menstrual status on lipid metabolism.

\subsection{Biochemical Analysis}

The serum samples were immediately refrigerated at $4^{\circ} \mathrm{C}$ or frozen at $-80^{\circ} \mathrm{C}$ until analysis. The serum glucose level was measured using a mutarotase-glucose oxidase method (Wako, Osaka, Japan). The serum fructose was enzymatically measured (Bioassay Systems, Hayward, CA, USA). The serum insulin was measured using a chemiluminescent 
enzyme immunoassay (Fujirebio, Tokyo). Insulin resistance was evaluated according to HOMA-IR [20]. The hemoglobin A1c (HbA1c) level was measured using a latex agglutination method (Fujirebio) and expressed as the National Glycohemoglobin Standardization Program (NGSP) value. The levels of free fatty acids (FFA) (Eiken Chemical, Tokyo) and lactate (Kyowa Medex, Tokyo) were measured enzymatically. For the assay of lactate, blood was immediately deproteinized. The levels of total ketone bodies (TKB), $\beta$-hydroxybutyrate $(\beta \mathrm{HB})$, and acetoacetate (AA) were measured using an enzymatic cycling method (Kainos, Tokyo). The level of total cholesterol (TC) was measured enzymatically (Sysmex, Kobe, Japan). The level of high-density lipoprotein-cholesterol (HDL-C) was measured by a direct method (Fujirebio), and we calculated the level of low-density lipoprotein-cholesterol (LDL-C) by using the Friedewald formula. The level of TG was enzymatically measured (Sekisui Medical, Tokyo). The level of remnant lipoprotein-cholesterol (RLP-C) was measured using a homogeneous assay (MetaboLead RemL-C ${ }^{\mathrm{TM}}$, Kyowa Medex, Tokyo). The level of apolipoprotein (apo) B was measured by an immunoturbidimetric method (Sekisui Medical). The level of apoB48 was measured by chemiluminescent enzyme immunoassay (Fujirebio). The concentration of apoB100 was calculated by subtracting the value of apoB48 from the value of apoB [13]. The apoE phenotype was measured using the isometric electrophoresis method (Phenotyping ApoE IEF System $^{\mathrm{TM}}$, Joko, Tokyo).

\subsection{Quantification of Postprandial Metabolism}

The postprandial changes in the concentrations of glucose, fructose, insulin, TG, RLP-C, apoB100, and apoB48 were calculated as the difference from the baseline mean value (as 0 at $0 \mathrm{~h}$ ) and are shown as $\Delta$ glucose, $\Delta$ fructose, $\Delta$ insulin, $\Delta \mathrm{TG}$, $\triangle R L P-C, \triangle a p o B 100$, and $\triangle a p o B 48$, respectively. We quantified the postprandial metabolism by calculating the incremental area under the curve ( $\triangle \mathrm{AUC})$, which was defined as the difference between the AUC determined by the trapezoidal method and the area below the baseline $(0 \mathrm{~h})$ from 0 to $6 \mathrm{~h}$, as described [1].

\subsection{Statistics}

We used SPSS ver. 19 software (IBM, Tokyo) for all the statistical analyses. Normal distribution of data was verified using the Shapiro-Wilk test for skewness and kurtosis of distribution. Non-normally distributed data were met for normal distribution when logarithmically transformed, and analyzed using parametric statistics. The data are presented as the mean \pm SEM. We analyzed the differences in the time course compared with the fasting values by performing a repeated measures analysis of variance (ANOVA), followed by the Dunnett test. The measured value differences at each time point in the 4 trials were assessed by a repeated measures ANOVA followed by the Bonferroni test. For all data, $p<0.05$ was considered statistically significant.

\section{Results}

The physical characteristics and fasting blood chemical data of the subjects are summarized in Table 1 . There were no significant differences in any of these data among the 4 trials (data not shown). The fasting and postprandial chemical data of glucose, fructose, insulin, lactate, and FFA in the 4 trials are presented in Table 2, and those of TG, RLP-C, apoB48, and apoB100 are presented in Table 3. The time courses of $\Delta$ glucose, $\Delta$ insulin, and $\Delta$ fructose are shown in Figure 1 , and those of $\Delta \mathrm{TG}, \Delta \mathrm{RLP}-\mathrm{C}, \Delta \mathrm{apoB} 48$, and $\Delta$ apoB100 are shown in Figure 2.

Table 1. Anthropometric and clinical characteristics of the subjects.

\begin{tabular}{llll}
\hline Age $($ years $)$ & 21.2 & \pm & 0.3 \\
Height $(\mathrm{cm})$ & 158.4 & \pm & 1.9 \\
Mass $(\mathrm{kg})$ & 52.6 & \pm & 1.9 \\
BMI $\left(\mathrm{kg} / \mathrm{m}^{2}\right)$ & 20.9 & \pm & 0.5 \\
Waist $(\mathrm{cm})$ & 69.4 & \pm & 2.2 \\
Hip $(\mathrm{cm})$ & 90.8 & \pm & 1.9 \\
W/H & 0.76 & \pm & 0.02 \\
VFA $\left(\mathrm{cm}^{2}\right)$ & 29.8 & \pm & 4.9 \\
SBP $(\mathrm{mmHg})$ & 108.8 & \pm & 1.7 \\
DBP $(\mathrm{mmHg})$ & 67.2 & \pm & 2.5 \\
PR (beats/min) & 73.9 & \pm & 3.5 \\
HbA1c $(\%)$ & 5.2 & \pm & 0.1 \\
HOMA-IR & 1.6 & \pm & 0.2 \\
TC $(\mathrm{mg} / \mathrm{dL})$ & 148.3 & \pm & 5.7 \\
HDL-C (mg/dL) & 55.0 & \pm & 1.7 \\
LDL-C (mg/dL) & 64.8 & \pm & 3.0 \\
\hline
\end{tabular}

SBP: systolic blood pressure, DBP: diastolic blood pressure, PR: pulse rate

\subsection{Glucose, Insulin, Fructose, Lactate, FFA, and Ketone Bodies}

In the FFr and FFrR trials, serum glucose concentration peaked at $0.5 \mathrm{~h}$, and returned to baseline at $1 \mathrm{~h}$ (Figure $1 \mathrm{~A}$ ). In the $\mathrm{F}$ and FR trials, the glucose concentration did not rise significantly during the experiment. The serum insulin concentration peaked at $0.5 \mathrm{~h}$ in all 4 trials (Figure 1B) and returned to baseline at $1 \mathrm{~h}$ in the $\mathrm{F}$ and FR trials and at $4 \mathrm{~h}$ in the FFr and FFrR trials. The $\triangle \mathrm{AUC}$ values in glucose and insulin in the FFr and FFrR trials were larger compared to the $\mathrm{F}$ and FR trials (data not shown).

The serum fructose concentration peaked at $0.5 \mathrm{~h}$ in the FFr trial and at $1 \mathrm{~h}$ in the FFrR trial, and returned to baseline at $4 \mathrm{~h}$ in both trials (Figure 1C). In the F and FR trials, the fructose concentration did not change during the experiment.

The concentration of lactate increased at $1 \mathrm{~h}$ in the FFr and FFrR trials. However, in the F and FR trials, no significant rise was observed. The serum FFA concentration was decreased at 1 and $2 \mathrm{~h}$ compared to each fasting level in the FFr and FFrR trials. In the F and FR trials, no decrease was observed. Thereafter, the concentration of FFA increased toward the end of the experiment in all trials.

In the F and FR trials, the serum TKB concentration increased at $1 \mathrm{~h}$, and further increased toward $6 \mathrm{~h}$. In the FFr and FFrR trials, TKB increased at 4 and $6 \mathrm{~h}$. $\beta \mathrm{HB}$ and AA concentrations showed similar patterns. (Data not shown) 
Table 2. Fasting and postprandial concentrations of glucose, fructose, insulin, lactate, and FFA.

\begin{tabular}{|c|c|c|c|c|c|c|c|}
\hline Time (h) & & $\mathbf{0}$ & 0.5 & 1 & 2 & 4 & 6 \\
\hline \multirow{4}{*}{$\begin{array}{l}\text { Glucose } \\
(\mathrm{mg} / \mathrm{dL})\end{array}$} & $\mathrm{F}$ & $86.4 \pm 1.9$ & $81.1 \pm 2.6^{*}$ & $80.6 \pm 2.7^{*}$ & $84.5 \pm 1.8$ & $82.8 \pm 2.1$ & $80.2 \pm 2.3^{*}$ \\
\hline & FR & $84.7 \pm 2.1$ & $84.6 \pm 2.1$ & $80.7 \pm 1.8$ & $84.2 \pm 2.3$ & $79.6 \pm 2.4^{*}$ & $75.8 \pm 2.3^{*}$ \\
\hline & FFr & $83.8 \pm 1.7$ & $92.9 \pm 3.0 *$ & $86.0 \pm 2.9$ & $85.2 \pm 1.3$ & $85.5 \pm 1.7$ & $82.7 \pm 1.1$ \\
\hline & FFrR & $86.6 \pm 1.2$ & $96.3 \pm 1.8^{*}$ & $91.0 \pm 2.1$ & $87.8 \pm 1.9$ & $84.0 \pm 1.9$ & $82.6 \pm 1.3$ \\
\hline \multirow{4}{*}{$\begin{array}{l}\text { Fructose } \\
(\mathrm{mg} / \mathrm{dL})\end{array}$} & $\mathrm{F}$ & $0.22 \pm 0.08$ & $0.22 \pm 0.09$ & $0.22 \pm 0.08$ & $0.22 \pm 0.09$ & $0.10 \pm 0.04$ & $0.09 \pm 0.05$ \\
\hline & FR & $0.09 \pm 0.06$ & $0.46 \pm 0.40$ & $0.27 \pm 0.19$ & $0.16 \pm 0.11$ & $0.12 \pm 0.07$ & $0.11 \pm 0.05$ \\
\hline & FFr & $0.19 \pm 0.06$ & $5.43 \pm 0.62 *$ & $4.93 \pm 0.58^{*}$ & $1.42 \pm 0.26^{*}$ & $0.10 \pm 0.06$ & $0.08 \pm 0.03$ \\
\hline & FFrR & $0.14 \pm 0.06$ & $4.59 \pm 0.66^{*}$ & $4.67 \pm 0.74 *$ & $1.79 \pm 0.21 *$ & $0.10 \pm 0.05$ & $0.13 \pm 0.06$ \\
\hline \multirow{4}{*}{$\begin{array}{l}\text { Insulin } \\
(\mu \mathrm{U} / \mathrm{mL})\end{array}$} & $\mathrm{F}$ & $7.5 \pm 1.0$ & $10.5 \pm 1.6^{*}$ & $9.3 \pm 1.4^{*}$ & $6.4 \pm 0.8$ & $4.2 \pm 0.7^{*}$ & $3.6 \pm 0.5^{*}$ \\
\hline & FR & $6.0 \pm 0.6$ & $9.3 \pm 1.3 *$ & $7.8 \pm 0.7$ & $4.9 \pm 0.5^{*}$ & $3.0 \pm 0.5^{*}$ & $2.5 \pm 0.3^{*}$ \\
\hline & FFr & $7.2 \pm 0.8$ & $19.0 \pm 2.3 *$ & $15.4 \pm 1.9 *$ & $9.0 \pm 0.9^{*}$ & $4.3 \pm 0.6$ & $3.7 \pm 0.4$ \\
\hline & FFrR & $7.2 \pm 0.6$ & $18.8 \pm 2.0^{*}$ & $14.2 \pm 1.3^{*}$ & $10.3 \pm 1.2$ & $4.5 \pm 0.4^{*}$ & $3.4 \pm 0.3^{*}$ \\
\hline \multirow{4}{*}{$\begin{array}{l}\text { Lactate } \\
(\mathrm{mg} / \mathrm{dL})\end{array}$} & $\mathrm{F}$ & $9.5 \pm 0.6$ & & $7.4 \pm 0.5$ & $8.7 \pm 0.5 *$ & $9.3 \pm 0.4$ & $8.7 \pm 0.3$ \\
\hline & FR & $8.4 \pm 0.8$ & & $15.0 \pm 1.6$ & $9.2 \pm 0.9$ & $7.3 \pm 0.5$ & $8.1 \pm 0.5$ \\
\hline & FFr & $9.9 \pm 1.1$ & & $13.2 \pm 1.1^{*}$ & $9.1 \pm 0.8$ & $8.7 \pm 0.7$ & $7.9 \pm 0.6$ \\
\hline & FFrR & $8.2 \pm 0.7$ & & $15.4 \pm 1.4^{*}$ & $8.0 \pm 0.9$ & $7.6 \pm 1.1$ & $7.4 \pm 0.7$ \\
\hline \multirow{4}{*}{$\begin{array}{l}\text { FFA } \\
(\mu \mathrm{mol} / \mathrm{L})\end{array}$} & $\mathrm{F}$ & $426.5 \pm 42.6$ & & $523.5 \pm 103.7$ & $545.4 \pm 77.5$ & $738.8 \pm 79.0 *$ & $886.5 \pm 114.9^{*}$ \\
\hline & FR & $548.8 \pm 77.3$ & & $610.7 \pm 186.3$ & $629.9 \pm 114.0$ & $726.3 \pm 46.0$ & $883.3 \pm 103.0$ \\
\hline & $\mathrm{FFr}$ & $492.5 \pm 40.7$ & & $201.8 \pm 19.9 *$ & $258.0 \pm 38.7$ & $586.2 \pm 36.6$ & $667.7 \pm 61.4 *$ \\
\hline & FFrR & $475.1 \pm 62.0$ & & $201.0 \pm 72.1 *$ & $181.3 \pm 38.5^{*}$ & $596.3 \pm 39.0$ & $720.4 \pm 60.4^{*}$ \\
\hline
\end{tabular}

${ }^{*} p<0.05$ compared to the fasting values. $\# p<0.05$ compared between the trials.
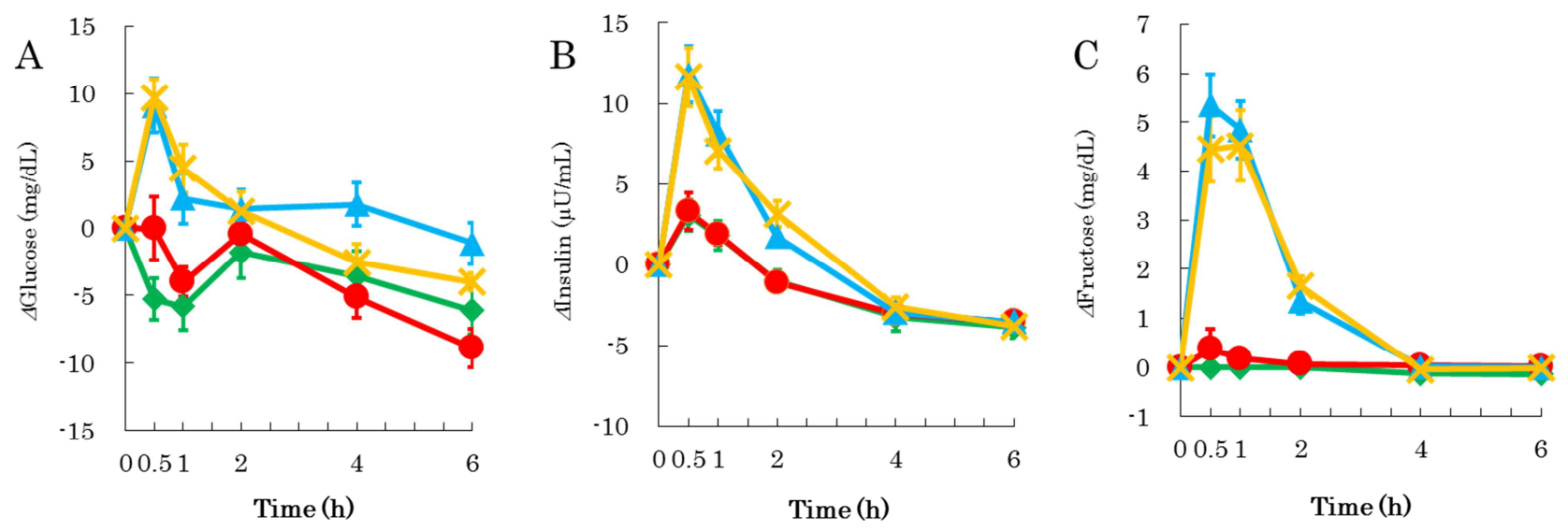

$\checkmark$ : F trial, : FR trial, $\Delta$ : FFr trial, $\times$ : FFrR trial

Figure 1. The time courses of glucose, insulin, and fructose.

\subsection{TG, RLP-C, apoB48, and apoB100}

The serum TG concentration peaked at $4 \mathrm{~h}$ in all 4 trials (Figure 2A). The rise in the TG concentration delayed in the FFr trial and especially in the FFrR trial. The peak TG values in the FFr and FFrR trials tended to be higher than those of the $\mathrm{F}$ and FR trials. The TG concentration returned to baseline at the end of the experiment in the F and FR trials, but not in the FFr and FFrR trials. The RLP-C concentration showed a similar pattern. RLP-C returned to baseline at the end of the experiment in the F and FR trials, but not in the FFr and FFrR trials (Figure 2B). The values at the end of the experiment were higher in the with-fructose trials (FFr and FFrR) compared to the without-fructose trials (F and FR). The rise in the RLP-C concentration was delayed in the FFr and FFrR trials compared to the F trial. In the FFr and FFrR trials, the $\triangle \mathrm{AUC}$ values for $\mathrm{TG}$ and RLP-C were larger (but not significantly so) compared to the F and FR trials (data not shown).

apoB48 is the structural protein of exogenous or intestinal lipoproteins, namely chylomicron and its remnant, and apoB48 is an index of the number of exogenous lipoprotein particles. apoB100 is the structural protein of endogenous or hepatic lipoproteins, namely very low-density lipoprotein (VLDL), VLDL remnant, and LDL, and apoB100 is an index of the number of endogenous lipoprotein particles. The rise in the serum apoB48 concentration was delayed in the FFrR trial compared to the $\mathrm{F}$ and FR trial (Figure 2C). The peaks in the FFr and FFrR trials were higher, but not significantly, than those in the F and FR trials. The apoB48 concentration did not return to baseline at the end of the experiment in all trials. The $\triangle \mathrm{AUC}$ for apoB48 was not significantly different among the 4 trials (data not shown). The apoB100 concentration increased toward the end of the experiment in all 4 trials (Figure 2D). 
Table 3. Fasting and postprandial concentrations of TG, RLP-C, apoB100, and apoB48.

\begin{tabular}{|c|c|c|c|c|c|c|c|c|c|}
\hline Time (h) & & $\mathbf{0}$ & 1 & & 2 & & 4 & 6 & \\
\hline \multirow{4}{*}{$\begin{array}{l}\text { TG } \\
(\mathrm{mg} / \mathrm{dL})\end{array}$} & $\mathrm{F}$ & $66.3 \pm 14.0$ & $68.4 \pm 13.8$ & & $78.1 \pm 14.4^{*}$ & & $81.7 \pm 16.3 *$ & $67.4 \pm 16.6$ & \\
\hline & FR & $62.3 \pm 11.7$ & $65.3 \pm 11.4$ & & $71.0 \pm 11.4^{*}$ & & $75.9 \pm 14.1 *$ & $59.2 \pm 12.2$ & \\
\hline & $\mathrm{FFr}$ & $65.7 \pm 8.1$ & $66.1 \pm 7.6$ & & $74.8 \pm 8.2$ & & $93.8 \pm 13.0^{*}$ & $82.9 \pm 11.8 *$ & \\
\hline & FFrR & $66.5 \pm 8.4$ & $67.7 \pm 8.3$ & & $71.0 \pm 7.7$ & & $91.3 \pm 10.6^{*}$ & $82.2 \pm 11.8 *$ & \\
\hline \multirow{3}{*}{$\begin{array}{l}\text { RLP-C } \\
(\mathrm{mg} / \mathrm{dL})\end{array}$} & $\mathrm{F}$ & $3.6 \pm 1.0$ & $3.6 \pm 0.9$ & & $4.3 \pm 0.9$ & & $4.7 \pm 1.1^{*}$ & $4.2 \pm 0.6$ & \\
\hline & FR & $3.4 \pm 0.9$ & $3.5 \pm 0.8$ & & $3.9 \pm 0.8$ & & $4.5 \pm 1.0^{*}$ & $3.7 \pm 1.0$ & \\
\hline & FFr & $3.4 \pm 0.5$ & $3.2 \pm 0.5$ & & $3.9 \pm 0.4$ & & $5.3 \pm 0.9 *$ & $4.9 \pm 0.8^{*}$ & $\pi$ \\
\hline \multirow{4}{*}{$\begin{array}{l}\text { apoB48 } \\
(\mathrm{mg} / \mathrm{L})\end{array}$} & $\mathrm{F}$ & $1.8 \pm 0.3$ & $2.2 \pm 0.3$ & 7 & $3.3 \pm 0.4 *$ & & $3.6 \pm 0.5^{*}$ & $2.9 \pm 0.5^{*}$ & \\
\hline & FR & $1.6 \pm 0.3$ & $2.0 \pm 0.2$ & $\#$ & $3.1 \pm 0.3 *$ & & $3.7 \pm 0.6^{*}$ & $2.6 \pm 0.4^{*}$ & \\
\hline & FFr & $1.9 \pm 0.3$ & $1.9 \pm 0.2$ & & $3.9 \pm 0.5 *$ & & $4.7 \pm 0.7 *$ & $3.5 \pm 0.4^{*}$ & \\
\hline & FFrR & $1.8 \pm 0.4$ & $1.5 \pm 0.3$ & 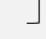 & $2.6 \pm 0.2$ & ]$^{*}$ & $5.4 \pm 0.8^{*}$ & $3.7 \pm 0.6^{*}$ & \\
\hline \multirow{3}{*}{$\begin{array}{l}\text { apoB100 } \\
(\mathrm{mg} / \mathrm{dL})\end{array}$} & $\mathrm{F}$ & $63.7 \pm 3.5$ & $62.9 \pm 3.5$ & & $63.3 \pm 3.7$ & & $63.3 \pm 3.7$ & $65.4 \pm 3.6^{*}$ & \\
\hline & FR & $63.8 \pm 4.6$ & $64.2 \pm 4.3$ & & $63.2 \pm 4.4$ & & $64.1 \pm 4.2$ & $65.4 \pm 4.6$ & \\
\hline & FFr & $65.8 \pm 3.6$ & $64.9 \pm 3.2$ & & $65.0 \pm 3.5$ & & $64.9 \pm 3.5$ & $66.5 \pm 3.7$ & \\
\hline
\end{tabular}

${ }^{*} \mathrm{p}<0.05$ compared to the fasting values. $\# \mathrm{p}<0.05$ compared between the trials.
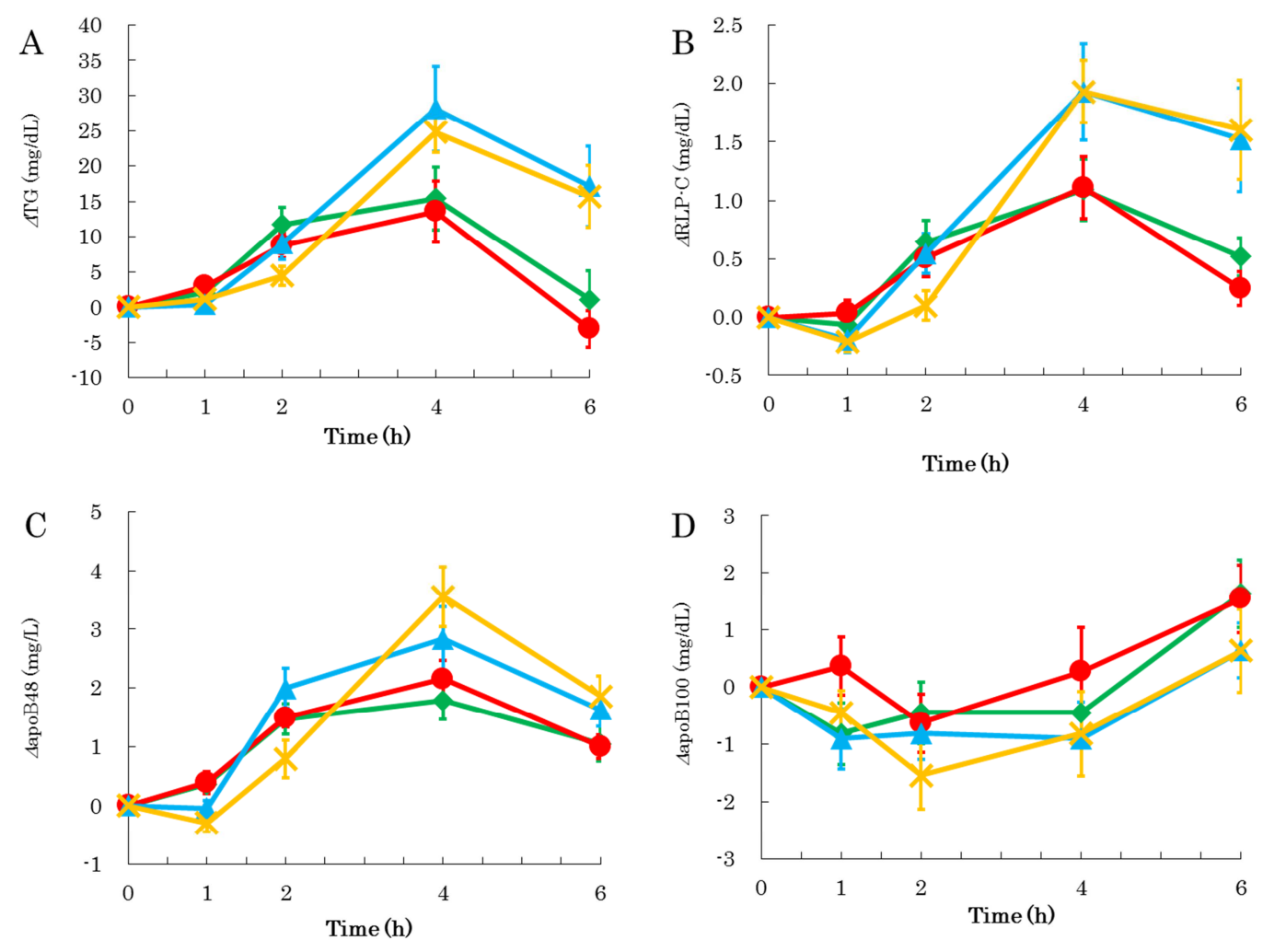

$\diamond:$ F trial, $\mathrm{O}$ : FR trial, $\Delta$ : FFr trial, $\times$ : FFrR trial

Figure 2. The time courses of TG, RLP-C, apoB48, and apoB100.

\section{Discussion}

RMD has been reported to be effective in ameliorating postprandial glycemia and lipidemia. The effects of RMD on postprandial lipidemia and glycemia induced by fat and starchy food (i.e., consisting of glucose, not fructose) have been studied. However, the absorption and metabolism of fructose is completely different from those of glucose. In the present study, RMD did not significantly inhibit fructose-induced postprandial hyperlipidemia in healthy young women.

Fructose may inhibit the absorption of fat and delay postprandial lipidemia [1]. Fructose is rapidly taken up into the liver without a feedback mechanism, and it may stimulate the synthesis of TG, leading to the formation of VLDL particles [21]. Fructose also delays the absorption of co-ingested fat and/or chylomicron formation and secretion. Chylomicron from the intestine will therefore compete for lipoprotein lipase with VLDL secreted from the liver, further delaying and exacerbating postprandial lipidemia.

We observed that the rise in the apoB48 concentration was delayed in the presence of RMD, and the peak value tended to be higher. The peaks in the presence of fructose (FFr and FFrR) 
were higher (but not significantly) than those in the absence of fructose (F and FR). These results suggest that the absorption of fat was delayed by RMD, but RMD did not ameliorate the peak or the $\triangle \mathrm{AUC}$ of apoB48. The $\triangle \mathrm{AUC}$ of apoB48 was not different between the without-fructose trials ( $\mathrm{F}$ and FR), and between the with-fructose trials (FFr and FFrR), indicating that RMD did not affect the exogenous lipoprotein clearance.

The rise in the TG concentration was delayed in the with-fructose trials (FFr and especially FFrR) compared to the without-fructose trials (F and FR). We have demonstrated that fructose delays the clearance of postprandial exogenous and endogenous lipoproteins [1]. In the present investigation, TG and RLP-C concentrations returned to baseline at $6 \mathrm{~h}$ in the trials without fructose (F and FR), but not in the trials with fructose (FFr and FFrR). RLP-C shows the cholesterol content in remnants, particularly in endogenous or hepatic-derived TG-rich remnants [22]. Our results show that RMD further slows the delayed clearance of postprandial lipoproteins induced by fructose. However, the apoB48 concentration did not return to baseline at the end of the experiment in any of the trials, suggesting that postprandial lipoprotein metabolism did not end even $6 \mathrm{~h}$ after ingestion, irrespective of the presence or absence of fructose and/or RMD. Our results therefore indicate that RMD may delay the production/secretion of endogenous lipoprotein (namely VLDL) in the postprandial phase.

In the $\mathrm{F}$ and FR trials, the insulin levels were slightly but significantly increased at $0.5 \mathrm{~h}$ without a rise in the glucose concentration, probably mediated by incretins. With fructose (FFr and FFrR), the glucose concentration peak did not differ with or without RMD, but at 4 and $6 \mathrm{~h}$, the glucose concentration was slightly lower in the presence of RMD, suggesting a possible effect of RMD on glucose absorption/metabolism.

This study has some limitations. The number of subjects was relatively small, and the results are largely non-significant. The results should thus be interpreted with caution. However, irrespective of these shortcomings, further studies are warranted.

\section{Conclusion}

Although RMD has been reported to be effective in ameliorating postprandial lipidemi and glycemia induced by fat and starchy food, it did not significantly inhibit fructose-induced postprandial hyperlipidemia in healthy young women.

\section{Acknowledgements}

This work was supported by JSPS KAKENHI grant (No. $15 \mathrm{~K} 00855)$ from the Ministry of Education, Culture, Sports, Science and Technology, Japan. We thank Mr Tatsuo Nishioka, Kyowa Medex Co, for his technical assistance in the measurement of RLP-C. We also thank Ms Mika Ochiai, Ms Momoka Saito, Ms Kazuna Taruta, and Ms Yuuna Hitomi for their technical cooperation.

\section{References}

[1] Saito H, Kagaya M, Suzuki M, Yoshida A, Naito M: Simultaneous ingestion of fructose and fat exacerbates postprandial exogenous lipidemia in young healthy Japanese women. J Atheroscler Thromb. 2013; 20: 591-600.

[2] Saito H, Kato M, Yoshida A, Naito M: The ingestion of a fructose-containing beverage combined with fat cream exacerbates postprandial lipidemia in young healthy women. $\mathrm{J}$ Atheroscler Thromb. 2015; 22: 85-94.

[3] Saito H, Kato M, Yoshida A, Naito M: The ingestion of high-fructose syrup-containing cola with a hamburger delays postprandial lipid metabolism in young healthy Japanese women. J Food Nutr Sci. 2015; 3: 139-146.

[4] Kato M, Yoshida A, Naito M: Fast food ingestion for lunch delays postprandial lipid metabolism in young women. J Food Nutr Sci. 2017; 5: 116-121.

[5] Chong MFF, Fielding BA, Frayn KN: Mechanisms for the acute effect of fructose on postprandial lipemia. Am J Clin Nutr. 2007; 85: 1511-1520.

[6] Ohkuma K, Matsuda I, Katta Y, Hanno Y: Pyrolysis of starch and its digestibility by enzymes-characterization of resistant maltodextrin. J Jpn Soc Starch Sci. 1990; 37: 107-114 (In Japanese).

[7] Rafter JJ, Eng VW, Furrer R, Medline A, Bruce WR: Effects of calcium and $\mathrm{pH}$ on the mucosal damage produced by deoxycholic acid in the rat colon. Gut. 1986; 27: 1320-1329.

[8] Nagengast FM, Hectors MP, Buys WA, Van Tongeren JH: Inhibition of secondary bile acid formation in the large intestine by lactulose in healthy subjects of two different age groups. Eur J Clin Invest.1988; 18: 56-61.

[9] Fastinger ND, Karr-Lilienthal LK, Spears JK, Swanson KS, Zinn KE, Nava GM, Ohkuma K, Kanahori S, Gordon DT, Fahey GC Jr: A novel resistant maltodextrin alters gastrointestinal tolerance factors, fecal characteristics, and fecal microbiota in healthy adult humans. J Am Coll Nutr. 2008; 27: 356-366.

[10] Livesey G, Tagami H: Interventions to lower the glycemic response to carbohydrate foods with a low-viscosity fiber (resistant maltodextrin): meta-analysis of randomized controlled trials. Am J Clin Nutr. 2009; 89: 114-125.

[11] Kishimoto Y, Oga H, Tagami H, Okuma K, Gordon DT: Suppressive effect of resistant maltodextrin on postprandial blood triacylglycerol elevation. Eur J Nutr. 2007; 46: 133-138.

[12] Ikeda I, Tamakuni K, Sakuma T, Ozawa R, Inoue N, Kishimoto Y: Resistant maltodextrin decreases micellar solubility of lipids and diffusion of bile salt micelles and suppresses incorporation of micellar fatty acids into Caco-2 cells. J Nutr Sci Vitaminol. 2016; 62: 335-340.

[13] Kishimoto Y, Yoshikawa Y, Miyazato S, Oga H, Yamada T, Tagami H, Hashizume C, Yamamoto K: Effect of resistant maltodextrin on digestion and absorption of lipids. J Health Sci. 2009; 55: 838-844.

[14] Kishimoto Y, Hayashi N, Yamada T, Yuba K, Yamamoto K: Favorable effect of resistant maltodextrin on postprandial blood glucose, insulin and triglyceride levels. Jpn Pharmacol Ther. 2009; 37: 277-283 (In Japanese, abstract in English). 
[15] Aliasgharzadeh A, Dehghan P, Gargari BP, Asghari-Jafarabadi $\mathrm{M}$ : Resistant dextrin, as a prebiotic, improves insulin resistance and inflammation in women with type 2 diabetes: a randomised controlled clinical trial. Br J Nutr. 2015; 113: 321-330.

[16] Hashizume C, Kishimoto Y, Kanahori S, Yamamoto T, Okuma $\mathrm{K}$, Yamamoto K: Improvement effect of resistant maltodextrin in humans with metabolic syndrome by continuous administration. J Nutr Sci Vitaminol. 2012; 58: 423-430.

[17] Asano M, Fukakura N, Odachi J, Kawaraya C, Nanba A, Yasuda N, Yamamoto E: Use of fast foods among young people. Jpn J Nutr Diet. 2003; 61: 47-54 (In Japanese, Abstract in English).

[18] Bansal S, Buring JE, Rifai N, Mora S, Sacks FM, Ridker PM: Fasting compared with nonfasting triglycerides and risk of cardiovascular events in women. JAMA. 2007; 298: 309-316.
[19] Nabeno Y, Fukuchi Y, Matsutani Y, Naito M: Influence of aging and menopause on postprandial lipoprotein responses in healthy adult women. J Atheroscler Thromb. 2007; 14: 142-150.

[20] Matthews DR, Hosker JP, Rudenski AS, Naylor BA, Treacher DF, Turner RC: Homeostasis model assessment: insulin resistance and beta-cell function from fasting plasma glucose and insulin concentrations in man. Diabetologia. 1985; 28: 412-419.

[21] Tappy L, Lê KA: Metabolic effects of fructose and the worldwide increase in obesity. Physiol Rev. 2010; 90: 23-46.

[22] Fujioka Y, Ishikawa Y: Remnant lipoproteins as strong key particles to atherogenesis. J Atheroscler Thromb. 2009; 16: $145-154$. 